\title{
GERMINAÇÃO DE SEMENTES E EMERGÊNCIA DE PLÂNTULAS DE PAU-SANTO: UMA ANÁLISE CRÍTICA DO USO DE CORRELAÇÃO ${ }^{1}$
}

\author{
DENISE GARCIA DE SANTANA², MARISTELA ROSÁLIA ANASTÁCIO³, \\ JULIA ARAÚJO DE LIMA ${ }^{4}$, MARCELA BUENO DE MATTOS ${ }^{5}$
}

\begin{abstract}
RESUMO - Pau-santo (Kielmeyera coriacea) é a espécie fornecedora de cortiça mais importante do Cerrado, porém apresenta alto grau de polimorfismo entre os indivíduos, o que pode afetar de forma distinta os testes de germinação de sementes e emergência de plântulas. Diante disso, os objetivos foram correlacionar características de germinação de sementes com a emergência de plântulas da espécie e quantificar o impacto da variabilidade entre os indivíduos na correlação e no teste de significância. Nos testes de germinação e emergência, o delineamento experimental foi o de blocos casualizados com sete tratamentos correspondentes aos indivíduos, com três repetições em parcelas compostas de 32 sementes. Dois critérios distintos de avaliação foram adotados, o de protrusão da radícula, no teste de germinação, e de plântula normal, no teste de emergência. Os indivíduos de $K$. coriacea apresentaram alta capacidade de germinação e emergência, com alto grau de incerteza, baixa sincronia e espalhamento em relação ao tempo médio, embora em ambos os testes os indivíduos tenham apresentado classificação distinta quanto a essas características. As correlações entre medidas de germinação e emergência com dados originais e com resíduos foram diferentes, mostrando que a variabilidade intrínseca do indivíduo interfere no resultado da medida. A significância associada à correlação apenas indicou que o valor encontrado não é zero e, portanto, não pode ser interpretado como relevante na associação entre as características da espécie.
\end{abstract}

Termos para indexação: Kielmeyera coriacea Mart., significância, resíduos, variabilidade intraespecífica.

\section{SEED GERMINATION AND SEEDLING EMERGENCE OF Kielmeyera coriacea: A CRITICAL ANALYSIS OF THE USE OF CORRELATION}

\begin{abstract}
Kielmeyera coriacea is the most important cork supplying species of the Brazilian Savannah but it shows a high degree of individual polymorphism which can affect seed germination tests and seedling emergence. The objectives of this study were to correlate seed germination characteristics with seedling emergence and quantify the impact of individual variability in the correlation and significance tests. A randomized block design was used in the germination and emergence tests with seven treatments corresponding to the individuals and three plot replications with 32 seeds each. Two distinct evaluation criteria were adopted: radicle protrusion in the germination test and a normal seedlings in the emergence test. $K$. coriacea individuals presented high germination and emergence capacities, with a high degree of uncertainty, low synchronization and spread in relation to average
\end{abstract}

${ }^{1}$ Submetido em 23/03/2009 Aceito para publicação em 19/03/2010.

${ }^{2}$ Eng. Agr., Dr., Professora Adjunta, Instituto de Ciências Agrárias, UFU, Caixa postal 593, 38400-902. Uberlândia, MG, e-mail: dgsantana@umuarama.ufu.br.

${ }^{3}$ Bióloga, Mestranda em Agronomia, Instituto de Ciências Agrárias, UFU, Caixa postal 593, 38400-902. Uberlândia, MG, e-mail: maristelaanastacio@yahoo.com.br.

${ }^{4}$ Bióloga, Doutoranda em Agronomia, Instituto de Ciências Agrárias, UFU, Caixa postal 593,38400-902. Uberlândia, MG, e-mail: juliaaraujo@yahoo. com.br.

${ }^{5}$ Eng. Agr., Instituto de Ciências Agrárias, UFU, Caixa postal 593, 38400902. Uberlândia, MG, mbmattos@yahoo.com.br. 
time, although in both tests the individuals showed a distinct classification with regards to these characteristics. The correlations between germination and emergence measurements with original data and with residuals were different, showing that the individual's intrinsic variability interferes with the measurement result. The significance associated with the correlation only indicated that the value found is not zero and, therefore, cannot be interpreted as being important in the association among the species characteristics.

Index terms: Kielmeyera coriacea, Mart., significance, residuals, intra-specific variability.

\section{INTRODUÇÃO}

O gênero Kielmeyera Mart. é endêmico da América do Sul, compreende cerca de 47 espécies, sendo 45 nativas do Brasil (Barros, 2002). Entre as espécies do gênero, Kielmeyera coriacea Mart. (pau-santo) distribuise amplamente pelo Brasil, desde o estado do Amazonas até São Paulo em áreas de campo sujo, cerrado e cerradão (Almeida et al., 1998). Os indivíduos possuem distribuição espacial agregada (Oliveira et al., 1989) e alta frequência em áreas de cerrado sensu stricto, entre 33 e 121 indivíduos por ha (Barreira et al., 2002; Rezende et al., 2005). A espécie é a mais importante fornecedora de cortiça do cerrado, em função da abundante produção de súber (Macedo, 1991); também é melífera (Brandão e Ferreira, 1991), ornamental (Macedo, 1991), além de apresentar atividade farmacológica (Martins et al., 2004).

Testes de polinização artificial revelaram que as espécies de Kielmeyera são xenogâmicas, com forte grau de auto-incompatibilidade de ação tardia, uma vez que os tubos polínicos resultantes de autopolinização são bloqueados na base do estilo ou entre os lóculos do ovário. Como conseqüência, a porcentagem de frutos resultantes de polinização cruzada é maior do que de polinização natural (Barros, 2002), com porcentagem elevada, em torno de $33 \%$, de sementes abortadas em Kielmeyera coriacea (Almeida et al., 1998).

A espécie apresenta alto grau de polimorfismo entre os indivíduos, o que pode afetar de maneira distinta os testes de germinação de sementes e emergência de plântulas e, consequentemente, a avaliação da qualidade fisiológica das sementes. Para determinar a qualidade fisiológica das sementes, o teste de germinação é o mais utilizado, sendo realizado em laboratório sob condições controladas de temperatura, substrato e luz, possibilitando que as sementes expressem o máximo poder germinativo sem que haja interferências externas indesejáveis (Piña-Rodrigues et al.,
2004). Por outro lado, o teste de emergência de plântulas não depende só da energia contida no endosperma ou nos cotilédones (Hackbart e Cordazzo, 2003), mas também das características físicas do substrato, como estrutura, aeração, capacidade de retenção de água e grau de infestação de patógenos (Albuquerque et al., 1998), além da temperatura, umidade, profundidade de semeadura e disponibilidade de oxigênio (Severino et al., 2004).

A relação entre dados obtidos em laboratório e em campo é normalmente quantificada pela correlação de Pearson, sendo bastante utilizada por tecnologistas de sementes para comparações entre metodologias de testes de vigor, especialmente em trabalhos incluindo emergência em campo. Esses experimentos são comuns com sementes de espécies cultivadas como soja (Shuab et al., 2002), melão (Bhering et al., 2004) e algodão (Dutra e Medeiros Filho, 2008), mas também com espécies florestais nativas como Mimosa caesalpiniifolia Benth (Alves et al., 2005), Hancornia speciosa Gomes (Barros et al., 2006), Schizolobium parahyba (Vell.) S.F. Blake (Cherobini, 2006) e Cedrela fissilis Vell (Cherobini et al., 2008).

Apesar da ampla aplicação, a correlação sofre diversas críticas. Marcos Filho et al. (1984) alertam para o fato de a medida ser apenas um indicativo da variação conjunta entre duas características, não podendo ser analisada isoladamente. Schield (1995) enfatiza que a busca de relações de causa e efeito entre as características com o uso da correlação, é um dos grandes equívocos de interpretação da medida. Segundo Miller (1994), os problemas se tornam maiores quando correlações baixas ou desprezíveis, segundo critério de Davis (1971), são discutidas como significantes.

Diante disso, os objetivos nessa pesquisa foram correlacionar características de germinação de sementes com a emergência de plântulas de pau-santo e quantificar o impacto da variabilidade entre os indivíduos na correlação e no teste de significância. 


\section{MATERIAL E MÉTODOS}

Frutos de sete indivíduos de Kielmeyera coriacea foram coletados, individualmente, em agosto de 2004 sendo que três indivíduos estão localizados no município

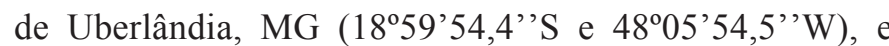
quatro no município de Cristianópolis, GO $\left(17^{\circ} 11^{\prime} 57^{\prime}\right.$ 'S e $48^{\circ} 42^{\prime} 21^{\prime} ' \mathrm{~W}$ ), identificados e debulhados manualmente para a retirada das sementes que, em seguida, foram homogeneizadas e as alas cortadas, sem comprometimento do embrião.

Para o estudo, dois experimentos independentes foram conduzidos simultaneamente, um em câmara de germinação, adotando-se a protrusão da radícula como critério de germinação, e outro em casa de vegetação, sendo o critério de avaliação a exposição dos cotilédones totalmente expandidos acima do substrato.

Em ambos os testes, o delineamento experimental foi o deblocos casualizados com sete tratamentos correspondentes aos indivíduos, com três repetições em parcelas compostas de 32 sementes cada. No teste de germinação, as sementes foram acondicionadas em caixas tipo gerbox, com tampa, com dimensões de $11,5 \mathrm{~cm} \times 11,5 \mathrm{~cm}$ x 3,5 cm, contendo $180 \mathrm{~mL}$ de vermiculita umedecida com $80 \mathrm{~mL}$ de água destilada, dispostas em câmara de germinação com médias de temperaturas mínima e máxima de $23,3 \pm 1,3{ }^{\circ} \mathrm{C}$ e de $26,2,7 \pm 1,0^{\circ} \mathrm{C}$, respectivamente. No teste de emergência, as sementes foram dispostas em bandejas multicelulares (128 células) de poliestireno expandido a $1 \mathrm{~cm}$ de profundidade contendo mistura de substrato comercial (Plantmax $\left.{ }^{\circledR}\right)$ e vermiculita (volume de expansão de $0,1 \mathrm{~m}^{3}$ ) na proporção 1:1. As bandejas foram mantidas em casa de vegetação, com médias de temperaturas mínima e máxima de $31,5 \pm 3,2{ }^{\circ} \mathrm{C}$ e de $36,7 \pm 1,8^{\circ} \mathrm{C}$, respectivamente, e regadas diariamente.

Das contagens diárias de sementes germinadas e plântulas emergidas determinaram-se as porcentagens de germinação e de emergência, tempo médio de germinação e de emergência (Labouriau, 1983), velocidade de germinação e de emergência (Maguire, 1962), coeficiente de variação do tempo de germinação e de emergência, que mede a distribuição do processo em torno do tempo médio (Ranal e Santana, 2006), incerteza de germinação e de emergência, medida que indica se o processo ocorreu ou não (Labouriau e Valadares, 1976) e sincronia de germinação e de emergência, que marca quando no mínimo duas sementes germinam ou emergem juntas (Primack, 1980). As medidas foram inicialmente testadas quanto às pressuposições de normalidade dos resíduos (teste de Shapiro-Wilk) e homogeneidade entre as variâncias (teste de Bartlett) e, em seguida, foi aplicada a análise de variância, testando-se a diferença entre os indivíduos pelo teste de Scott-Knott. Em todos os testes, utilizou-se $\alpha=0,05$ como o valor de significância.

Correlações para uma mesma medida obtida em câmara de germinação ou em casa de vegetação foram calculadas para os dados originais e para os resíduos padronizados. $\mathrm{Na}$ padronização, foram considerados os resíduos do modelo de análise de variância de um delineamento de blocos casualizados, propostos por Santana e Ranal (2006), segundo a expressão $d_{i j}=\left(y_{i j}-\bar{y}_{i .}-\bar{y}_{. j}+\bar{y}_{. .}\right)$onde $y_{i j}$ é o valor da variável $Y$, que recebeu o tratamento $i$ no bloco $j ; \bar{y}_{i .}$, valor médio da variável $Y$, no tratamento $i ; \bar{y}_{. j}$, valor médio da variável $Y$, no bloco $j$ e $\bar{y}$. média geral da variável $Y$. Tanto correlações com dados originais quanto com os resíduos foram testadas pelo $t$ de "Student" a 0,05 de significância e as magnitudes foram acompanhadas dos adjetivos propostos por Davis (1971), sendo $r=1$ correlação perfeita; $0,70 \leq r \leq 0,99$ muito alta; $0,50 \leq r \leq$ 0,69 substancial; $0,30 \leq r \leq 0,49$ moderada; $0,10 \leq r \leq 0,29$ baixa e $0,01 \leq r \leq 0,09$ desprezível.

\section{RESULTADOS E DISCUSSÃO}

A classificação do teste de Scott-Knott indicou maior formação de grupos entre os indivíduos de pau-santo no teste de germinação de sementes, quando comparada à formação encontrada no teste de emergência de plântulas (Tabela 1). A diferença entre a maior e a menor média foi de $53,12 \%$ para a germinação das sementes e de $41,67 \%$ para a emergência de plântulas. Essa grande amplitude revelou alta variabilidade entre os indivíduos, podendo justificar, em parte, as diferenças encontradas na literatura referentes à capacidade de germinação da espécie, variável entre $60 \%$ e $90 \%$ obtida por vários autores (Melo et al., 1979; Botelho e Carneiro, 1992; Coelho et al., 1997). Não há registros de dormência em sementes da espécie e as baixas porcentagens de germinação obtidas para sementes dos indivíduos 4 e 7 ocorreram devido à deterioração, uma vez que ao final do testes todas estavam mortas.

Os indivíduos foram separados pelos seus tempos médios em três grupos distintos, tanto para a germinação quanto para a emergência (Tabela 1), sendo gastos entre 2,76 e 7,68 dias para que ocorresse a protrusão da radícula e entre 11,69 a 20,74 dias para a emergência da plântula, sendo as sementes do indivíduo 5 as mais rápidas e as do indivíduo 7 as mais lentas, em ambos os testes. Na literatura, 
o tempo de germinação da espécie foi estimado em 9,8 dias por Coelho et al. (1997) e em 12 dias por Oliveira et al.
(2008), ambos para a protrusão da radícula e 14 dias para emergência da plântula (Oliveira et al., 2008).

TABELA 1. Medidas de germinação de sementes e emergência de plântulas de Kielmeyera coriacea Mart. obtidas de sete indivíduos distribuídos no município de Uberlândia, MG e município de Cristianópolis, GO.

\begin{tabular}{|c|c|c|c|c|c|c|c|c|}
\hline \multirow{2}{*}{ Medida (unidade) ${ }^{1}$} & \multirow{2}{*}{ critério } & \multicolumn{7}{|c|}{ Indivíduos } \\
\hline & & 1 & 2 & 3 & 4 & 5 & 6 & 7 \\
\hline \multirow{2}{*}{$G(\%)$} & germinação & $100,00 \mathrm{a}$ & $82,29 \mathrm{~b}$ & $98,96 \mathrm{a}$ & $46,88 \mathrm{~d}$ & $88,54 \mathrm{~b}$ & $89,58 \mathrm{~b}$ & $59,38 \mathrm{c}$ \\
\hline & emergência & 97,92 a & 83,33 a & $97,92 \mathrm{a}$ & $73,96 \mathrm{~b}$ & $93,75 \mathrm{a}$ & $93,75 \mathrm{a}$ & $56,25 \mathrm{c}$ \\
\hline \multirow{2}{*}{$\bar{t}(\mathrm{dia})$} & germinação & $3,95 \mathrm{~b}$ & $3,54 \mathrm{a}$ & $4,61 \mathrm{~b}$ & $4,42 \mathrm{~b}$ & $2,76 \mathrm{a}$ & $4,35 \mathrm{~b}$ & $7,68 \mathrm{c}$ \\
\hline & emergência & $14,84 \mathrm{~b}$ & $15,29 \mathrm{~b}$ & $15,14 b$ & $12,19 \mathrm{a}$ & $11,69 \mathrm{a}$ & $15,70 \mathrm{~b}$ & $20,74 \mathrm{c}$ \\
\hline \multirow{2}{*}{$\begin{array}{c}V E \\
\left(\text { sementes ou plântulas } \text { dia }^{-1} \text { ) }\right.\end{array}$} & germinação & $9,17 \mathrm{~b}$ & $8,48 \mathrm{~b}$ & $7,30 \mathrm{~b}$ & $3,93 \mathrm{c}$ & $11,99 \mathrm{a}$ & $7,96 \mathrm{~b}$ & $2,77 \mathrm{c}$ \\
\hline & emergência & $2,25 \mathrm{~b}$ & $1,85 \mathrm{~b}$ & $2,13 \mathrm{~b}$ & $2,00 \mathrm{~b}$ & $2,70 \mathrm{a}$ & $2,05 \mathrm{~b}$ & $0,93 \mathrm{c}$ \\
\hline \multirow{2}{*}{$C V_{t}(\%)$} & germinação & $29,08 \mathrm{a}$ & $41,08 \mathrm{a}$ & $25,40 \mathrm{a}$ & $45,41 \mathrm{a}$ & $42,32 \mathrm{a}$ & $56,01 \mathrm{a}$ & $38,20 \mathrm{a}$ \\
\hline & emergência & $24,31 \mathrm{a}$ & 24,87 a & $17,52 \mathrm{a}$ & $17,95 \mathrm{a}$ & $26,12 \mathrm{a}$ & $27,68 \mathrm{a}$ & $20,31 \mathrm{a}$ \\
\hline \multirow{2}{*}{$I$ (bit) } & germinação & $2,12 \mathrm{a}$ & $2,02 \mathrm{a}$ & $1,99 \mathrm{a}$ & $1,80 \mathrm{a}$ & $1,87 \mathrm{a}$ & $2,46 \mathrm{~b}$ & $2,65 \mathrm{~b}$ \\
\hline & emergência & $3,30 \mathrm{a}$ & $2,83 \mathrm{a}$ & $2,71 \mathrm{a}$ & $2,75 \mathrm{a}$ & $2,71 \mathrm{a}$ & $3,21 \mathrm{a}$ & $2,30 \mathrm{a}$ \\
\hline \multirow{2}{*}{$Z$} & germinação & $0,24 \mathrm{a}$ & $0,28 \mathrm{a}$ & $0,29 \mathrm{a}$ & $0,30 \mathrm{a}$ & $0,32 \mathrm{a}$ & $0,20 \mathrm{a}$ & $0,14 \mathrm{a}$ \\
\hline & emergência & $0,08 \mathrm{~b}$ & $0,15 \mathrm{a}$ & $0,18 \mathrm{a}$ & $0,13 \mathrm{a}$ & $0,15 \mathrm{a}$ & $0,09 \mathrm{~b}$ & $0,07 \mathrm{~b}$ \\
\hline
\end{tabular}

${ }^{1}$ Médias seguidas por letras distintas na linha diferem entre si pelo de Scott-Knott a 0,05 de significância; $G$ : percentual de sementes germinadas ou plântulas emergidas; $\bar{t}$ : tempo médio; $V E$ : velocidade de emergência; $C V_{\mathrm{t}}$ : coeficiente de variação do tempo; I: Incerteza; $Z$ : sincronia.

Com a mesma quantidade de grupos formados entre germinação e emergência, porém com os indivíduos classificados de maneiras distintas, a velocidade de emergência registrou valores de $V E$ entre 2,77 e 11,99 sementes germinadas por dia, para o critério protrusão, e entre 0,93 e 2,70 plântulas por dia, para o critério de emergência (Tabela 1). Da mesma forma que o tempo médio, sementes e plântulas do indivíduo 5 foram consideradas de germinação e de emergência rápidas e as do indivíduo 7 consideradas lentas.

Quanto aos coeficientes de variação do tempo, tanto para a germinação quanto para a emergência, não foram constatadas diferenças estatísticas entre os indivíduos (Tabela 1). Para a incerteza houve a formação de dois grupos para a germinação, porém não foi detectada diferença significativa na emergência das plântulas dos indivíduos para esta característica. Na sincronia, o resultado foi inverso; formação de dois grupos no teste de emergência, sem formação na germinação. Coeficientes de variação do tempo acima de $20 \%$, para a maioria dos indivíduos, valores de $I$ acima de zero $(I \geq 1,80)$ e de $Z$ próximos à zero $(Z \leq 0,32)$ revelaram processos de germinação e de emergência de pausanto espalhados em relação ao tempo médio, com alto grau de incerteza e baixa sincronia (Tabela 1).

De maneira geral, as classificações dos indivíduos quanto à capacidade, ao tempo, à velocidade, à uniformidade, à incerteza e à sincronia foram distintas no teste de germinação em relação ao teste de emergência, ainda que o número de grupos formados segundo o teste de Scott-Knott tenha sido o mesmo para algumas características em ambos os testes (Tabela 1). Assim, é de se esperar que, para todas as características, as correlações entre germinação e emergência sejam, no máximo, moderadas (sensu Davis, 1971).

Na Tabela 2, correlação muito alta (sensu Davis, 1971) entre porcentual de germinação e emergência de plântulas de pau-santo, usando dados originais, parece superestimar a relação que os testes apresentaram entre si. Por outro lado, a mesma associação obtida com resíduos padronizados, indica não haver correlação entre os testes $(P \geq 0,05)$. A superestimação da correlação com dados originais se deve 
aos efeitos não padronizados dos indivíduos, previstos por Santana e Ranal (2006). Para tempo médio e velocidade, os resultados foram similares sendo encontradas correlações muito altas entre os testes para ambas as características, usando dados originais, e correlações apenas moderadas com os resíduos (Tabela 2).

TABELA 2. Coeficiente de correlação linear de Pearson $(r)$ entre os testes de germinação de sementes recém-colhidas e plântulas emergidas provenientes de sementes de Kielmeyera coriacea coletadas de sete indivíduos distribuídos no município de Uberlândia, MG e município de Cristianópolis, GO.

\begin{tabular}{lcc}
\hline \multicolumn{1}{c}{ Medida } & \multicolumn{2}{c}{ Correlação } \\
\cline { 2 - 3 } & \multicolumn{1}{c}{ dados originais } & resíduos \\
\hline$G:$ germinação e emergência & $0,79(0,0000)^{1}$ & $0,25(0,1324)^{1}$ \\
$\bar{t}:$ tempo médio de germinação e emergência & $0,83(0,0000)$ & $0,38(0,0432)$ \\
$V E:$ velocidade de emergência & $0,75(0,0001)$ & $-0,41(0,0337)$ \\
$C V_{\mathrm{t}}:$ coeficiente de variação do tempo & $0,05(0,4160)$ & $-0,45(0,0197)$ \\
$I:$ incerteza & $-0,26(0,1274)$ & $-0,44(0,0242)$ \\
$Z:$ sincronia & $0,26(0,1220)$ & $-0,49(0,0115)$ \\
\hline
\end{tabular}

${ }^{1}$ Os valores entre parênteses representam probabilidades e, quando menores que 0,05 ; indicam que o valor da correlação não é zero.

Correlações não significativas $(\mathrm{P} \geq 0,05)$ entre teste de germinação e na emergência para coeficiente de variação do tempo, incerteza e sincronia com o uso dos dados originais, passaram a ser significativas, ainda que moderadas, com o uso de resíduos. Destaca-se, portanto, que o uso dos dados originais pode também subestimar a associação entre variáveis (Santana e Ranal, 2006).

A significância do teste de correlação (Tabela 2) não pode ser interpretada da maneira usual feita para a significância do teste $F$ da análise de variância, uma vez que os testes apresentam hipóteses distintas, embora muitos desconheçam a hipótese envolvida na medida de correlação. Quando pesquisadores apresentam a significância associada à correlação e esta é significativa, a única inferência que pode ser feita é que o valor encontrado não é zero (Miller, 1994), o que não implica, necessariamente, que seja relevante. $\mathrm{Na}$ Tabela 2, a correlação significativa $(\mathrm{P} \geq 0,05)$ para tempo médio, usando resíduos, é apenas moderada (sensu Davis, 1971), da mesma maneira em que é para coeficiente de variação do tempo, incerteza e sincronia.

As discussões apresentadas para a comparação entre indivíduos de pau-santo podem ser estendidas para todos os experimentos nos quais a variável sofre os efeitos dos lotes, das diferentes metodologias empregadas ou qualquer outro tratamento envolvido. Neste contexto, correlações entre os resultados de testes realizados em laboratório e em campo, entre os de laboratório e os de vigor, devem ser aplicadas e interpretadas com cautela, e nunca de forma isolada, como sugeriram Marcos Filho et al. (1984).

\section{CONCLUSÕES}

As sementes de diferentes indivíduos de $K$. coriacea podem apresentar alta capacidade de germinação e de emergência, com alto grau de incerteza, baixa sincronia e espalhamento em relação ao tempo médio, embora em ambos os testes, os indivíduos possam apresentar classificação distinta quanto a essas características.

As correlações entre medidas de germinação e de emergência de plântulas com dados originais e com resíduos podem ser diferentes, mostrando que a variabilidade intrínseca do indivíduo interfere no resultado da medida.

\section{AGRADECIMENTOS}

À Coordenação de Aperfeiçoamento de Pessoal de Nível Superior (CAPES), pela concessão da bolsa de mestrado da segunda autora; à Fundação de Amparo à Pesquisa do Estado de Minas Gerais (FAPEMIG), pela concessão da bolsa de doutorado da terceira autora.

\section{REFERÊNCIAS}

ALBUQUERQUE, M.C.F.; RODRIGUES, T.J.D.; MINOHARA, L.; TEBALDI, N.D.; SILVA, L.M. de M. Influência da temperatura e do substrato na germinação 
de sementes de saguaraji (Colubrina glandulosa Perk. Rhamnaceae). Revista Brasileira de Sementes, v.20, n.2, p.108-111, 1998.

ALMEIDA, S.P.; PROENÇA, C.E.B.; SANO, S.M.; RIBEIRO, J.F. Cerrado: espécies vegetais úteis. Planaltina: EMBRAPA/CPAC, 1998. 464p.

ALVES, E.U.; BRUNO, R.L.A.; OLIVEIRA, A.P.; ALVES, A.U.; ALVES, A.U.; PAULA, R.C. de. Influência do tamanho e da procedência de sementes de Mimosa caesalpiniifolia Benth. sobre a germinação e vigor. Revista Árvore, v.29, n. 6, p.877-885, 2005.

BARREIRA, S.; SCOLFORO, J.R.S.; BOTELHO, S.A.; MELLO, J.M. Estudo da estrutura da regeneração natural e da vegetação adulta de um cerrado sensu stricto para fins de manejo florestal. Scientia Forestalis, v.61, p.64-78, 2002.

BARROS, D.I.; BRUNO, R.L.A.; NUNES, H.V.; SILVA, G.C.; PEREIRA, W.E.; MENDONÇA, R.M.N. Métodos de extração de sementes de mangaba visando à qualidade fisiológica. Revista Brasileira de Fruticultura, v.28, n.1, p.25-27, 2006.

BARROS, M.A.G. Floração sincrônica e sistemas reprodutivos em quatro espécies de Kielmeyera Mart. (Guttiferae). Acta Botanica Brasilica, v.16, n.1, p.113-122, 2002.

BHERING, M.C.; DIAS, D.C.F.S.; TOKUHISA, D.; DIAS, L.A.S. Avaliação do vigor de sementes de melão pelo teste de deterioração controlada. Revista Brasileira de Sementes, v.26, n.1, p.125-129, 2004.

BOTELHO, S.A.; CARNEIRO, J.G.A. Influência da umidade, embalagens e ambientes sobre a viabilidade e vigor de sementes de pau-santo (Kielmeyera coriacea Mart.). Revista Brasileira de Sementes, v.14, n.1, p.41-46, 1992.

BRANDÃO, M.; FERREIRA, P.B.D. Flora apícola do cerrado. Informe Agropecuário, v.15, n.168, p.7-14, 1991.

CHEROBINI, E.A.I. Avaliação da qualidade de sementes e mudas de espécies florestais nativas. 2006. 115f. Dissertação (Mestrado em Engenharia Florestal) - Universidade Federal de Santa Maria - RS, 2006.

CHEROBINI, E.A.I.; MUNIZ, M.F.B.; BLUME, E. Avaliação da qualidade de sementes e mudas de cedro. Ciência Florestal, v.18, n.1, p.65-73, 2008.

COELHO, M.F.B.; ALBUQUERQUE, M.C.F.; DOMBROSKI, J.L.D.; FERRONATO, A. Germinação de sementes de plantas medicinais nativas e espontâneas do cerrado de Mato Grosso. In: LEITE, L.L.; SAITO, C.H. (Ed.). Contribuição ao conhecimento ecológico do cerrado. Brasília: UnB/ECL, 1997. p.75-78.

DAVIS, J. A. Elementary survey analysis. Englewood: Prentice-Hall, 1971. 206p.

DUTRA, A.S.; MEDEIROS FILHO, S. Teste de deterioração controlada na determinação do vigor em sementes de algodão. Revista Brasileira de Sementes, v.30, n.1, p.19-23, 2008.

HACKBART, V.C.S.; CORDAZZO, C.V. Ecologia das sementes e estabelecimento das plântulas de Hydrocotyle bonariensis Lam. Atlântica, v.25, n.1, p.61-65, 2003.

LABOURIAU, L.G. A germinação das sementes. Organização dos Estados Americanos. Programa Regional de Desenvolvimento Científico e Tecnológico. Série de Biologia. Monografia 24, 1983. 174p.

LABOURIAU, L.G.; VALADARES, M.E.B. On the germination of seeds of Calotropis procera (Ait.) Ait. f. Anais da Academia Brasileira de Ciências, v.48, n.2, p.263-284, 1976.

MACEDO, J.F. Plantas corticosas do cerrado e sua utilização. Informe Agropecuário, v.15, n.168, p.33-37, 1991.

MAGUIRE, J.D. Speed of germination - aid in selection and evaluation for seedling emergence and vigor. Crop Science, v.2, p.176-177, 1962.

MARCOS FILHO, J.; PESCARIN, H.M.C.; KOMATSU, Y.H.; DEMETRIO, C.G.B.; FANCELLI, A.L. Testes para avaliação do vigor de sementes de soja e sua relação com a emergência das plântulas em campo. Pesquisa Agropecuária Brasileira, v.19, n.5, p.605-13, 1984.

MARTINS, J.V.C.; OTOBONE, F.J.; SELA, V.R.; OBICI, S.; TROMBELLI, M.A.; CORTEZ, D. A.G.; AUDI, E.A. Activity of hydroethanolic extract from Kielmeyera coriacea stems on central nervous system in rats. Acta Scientiarum Health Sciences, v.26, n.2, p.365-368, 2004.

MELO, J.T.; RIBEIRO, J.F.; LIMA, V.L.G.F. Germinação de sementes de algumas espécies arbóreas nativas do cerrado. Revista Brasileira de Sementes, v.1, n.2, p.76-81, 1979.

MILLER, L.E. Correlations: description or inference? Journal of Statistical Education, v.35, n.1, p.5-7, 1994.

OLIVEIRA, L.Z.; CESARINO, F.; PANTOJA, T.F.; SILVA, B.M.S.; MORO, F.V. Caracterização morfológica de sementes, germinação e plântula de Kielmeyera coriacea (Spreng.) Mart. (Guttiferae). In: ENCONTRO LATINO AMERICANO DE PÓS-GRADUAÇÃO, 8., 2008, São José dos Campos. Anais... São José dos Campos: ESS/UFRJ, 2008, p.1-4.

OLIVEIRA, P.E.A.M.; RIBEIRO, J.F.; GONZALES, M.I. 
Estrutura e distribuição espacial de uma população de Kielmeyera coriacea Mart. de cerrados de Brasília. Revista Brasileira de Botânica, v.12, p.39-47, 1989.

PIÑA-RODRIGUES， F.C.M.; FIGLIOLIA， M.B.; PEIXOTO, M.C. Testes de qualidade In: FERREIRA, F.G.; BORGHETTI, F. (Ed.) Germinação: do básico ao aplicado. Porto Alegre: Artmed, 2004. p.283-297.

PRIMACK, R.B. Variation in the phenology of natural populations of montane shrubs in New Zealand. Journal of Ecology, v.68, n.3, p.849-862, 1980.

RANAL, M.A.; SANTANA, D.G. How and why to measure the germination process? Revista Brasileira de Botânica, v.29, n.1, p.1-11, 2006.

REZENDE，A.V.; SANQUETTA， C.R.; FIGUEIREDO FILHO, A. Efeito do desmatamento no estabelecimento de espécies lenhosas em um cerrado sensu stricto. Floresta, v.35, n.1, p.69-88, 2005.
SANTANA, D.G.; RANAL, M.A. Linear correlation in experimental design models applied to seed germination. Seed Science and Technology, v.34, p.233-239, 2006.

SCHIELD, M. Correlation, determination and causality in introductory statistics. Proceedings of the Section on Statistical Education, p.1-6, 1995.

SCHUAB, S.R.P.; BRACCINI, A.L.; FRANÇA NETO, J.B.; SCAPIM, C.A.; MESCHEDE, D.K. Utilização da taxa decrescimento das plântulas na avaliação do vigor de sementes de soja. Revista Brasileira de Sementes, v.24, n.2, p.90-95, 2002.

SEVERINO, L.S.; GUIMARÃES, M.M.B.; COSTA, F.X.; LUCENA, A.M.A.; BELTRÃO, N. E. de M.; CARDOSO, G.D. Emergência da plântula e germinação de semente de mamona plantada em diferentes posições. Revista de Biologia e Ciências da Terra, v.5, n.1, p.1-6, 2004. 